\title{
Filmographies
}

With a few exceptions, the following filmographies relate to the directorial careers of the four in this study, in particular regard to their 1940 s films. Other films are listed but mostly without detailed credits.

\section{Leslie Arliss}

As screenwriter or author of story only

1932 Tonight's the Night

The Innocents of Chicago

Strip! Strip! Hooray!!! (short)

Josser on the River

Holiday Lovers

1934 Jack Ahoy!

Orders Is Orders

My Old Dutch

Road House

1935 Heat Wave

1936 Rhodes of Africa

Where There's a Will

Everybody Dance

Windbag the Sailor

1937 Good Morning, Boys

Said O'Reilly to McNab

1939 Too Dangerous to Live

Come On George!

1940 The Second Mr Bush

For Freedom

Pastor Hall

Bulldog Sees It Through

1941 South American George 
1942 The Foreman Went to France

1943 The Saint Meets the Tiger

1953 Top of the Form

As director (other functions are included)

The Farmer's Wife, 1940, 82 mins

Directors: Norman Lee, Leslie Arliss

Production Company: Associated British

Producer: Walter C. Mycroft

Screenplay: Lee, Arliss, J.E. Hunter

Cinematography: Claude Friese-Greene

Editing: Flora Newton

Art Direction: Charles Gilbert

Music: Guy Jones

Main Cast: Basil Sydney (Samuel Sweetland), Wilfrid Lawson (Churdles Ash), Nora Swinburne (Araminta Grey), Patricia Roc (Sibley), Michael Wilding (Richard Coaker), Enid Stamp-Taylor (Mary Hearne), Betty Warren (Louisa Windeatt)

The Night Has Eyes, 1942, 81 mins

Director: Leslie Arliss

Production Company: Associated British

Producer: John Argyle

Screenplay: Argyle, Arliss, Alan Kennington (from his novel)

Cinematography: Günther Krampf

Editing: Flora Newton

Art Direction: Duncan Sutherland

Costume Design: Jill Casson

Music: Charles Williams

Main cast: James Mason (Stephen Deremid), Wilfrid Lawson (Jim Sturrock), Mary Clare (Mrs Ranger), Joyce Howard (Marian Ives), Tucker McGuire (Doris), John Fernald (Dr Barry Randall), Amy Dalby (Miss Miggs), Dorothy Black (Miss Fenwick)

The Man in Grey, 1943, 116 mins

Director: Leslie Arliss

Production Company: Gainsborough

Producer: Edward Black 
Screenplay: Arliss, Margaret Kennedy, Doreen Montgomery (from a novel by Eleanor Smith)

Cinematography: Arthur Crabtree

Editing: R.E. Dearing

Art Direction: Walter Murton

Costume Design: Elizabeth Haffenden

Music: Cedric Mallabee, Louis Levy

Main cast: Margaret Lockwood (Hesther Shaw), Phyllis Calvert (Clarissa Marr), James Mason (Lord Rohan), Stewart Granger (Peter Rokeby), Harry Scott (Toby), Martita Hunt (Miss Patchett), Helen Haye (Lady Rohan), Beatrice Varley (Gypsy), Raymond Lovell (Prince Regent), Nora Swinburne (Mrs Fitzherbert)

Love Story, 1944, 108 mins

Director: Leslie Arliss

Production Company: Gainsborough

Producer: Harold Huth

Screenplay: Arliss, Doreen Montgomery, Rodney Ackland

Cinematography: Bernard Knowles

Editing: A. Charles Knott

Art Direction: John Bryan

Costume Design: Elizabeth Haffenden

Music: Hubert Bath, Louis Levy

Main cast: Margaret Lockwood (Lissa Campbell), Stewart Granger (Kit Firth), Patricia Roc (Judy), Tom Walls (Tom Tanner), Reginald Purdell (Albert), Walter Hudd (Ray), A.E. Matthews (Col. Pitt Smith), Beatrice Varley (Miss Rossiter), Moira Lister (Carol)

The Wicked Lady, 1945, 104 mins

Director: Leslie Arliss

Production Company: Gainsborough

Producer: R.J. Minney

Screenplay: Arliss, Aimée Stuart, Gordon Glennon (from a novel by Magdalen King-Hall)

Cinematography: Jack Cox

Editing: Terence Fisher

Art Direction: John Bryan

Costume Design: Elizabeth Haffenden

Music: Louis Levy

Main cast: Margaret Lockwood (Barbara Worth), James Mason (Capt. Jerry Jackson), Patricia Roc (Caroline), Griffith Jones (Sir Ralph Skelton), Michael Rennie (Kit Locksby), Felix Aylmer (Hogarth), Enid Stamp-Taylor (Lady Henrietta Kingsclere), Francis Lister (Lord Kingsclere), Jean Kent 
(Jackson's doxy), Martita Hunt (Cousin Agatha), Beatrice Varley (Aunt Moll), Amy Dalby (Aunt Doll), Emrys Jones (Ned Cotterill).

\section{A Man About the House, 1947, 99 mins}

Director: Leslie Arliss

Production Company: British Lion

Producer: Edward Black

Screenplay: Arliss, J.B. Williams, John Perry (from a novel by Francis Brett Young and play by Perry)

Cinematography: Georges Périnal

Editing: Russell Lloyd

Costume Design: Georges K. Benda

Music: Nicholas Brodszky

Main cast: Margaret Johnston (Agnes Isit), Dulcie Gray (Ellen Isit), Kieron Moore (Salvatore), Guy Middleton (Sir Benjamin Dench), Felix Aylmer (Richard Sanctuary), Lillian Braithwaite (Mrs Armitage), Jone Salinas (Maria), Marisa Finiani (Assunta)

Idol of Paris, 1948, 105 mins

Director: Leslie Arliss

Production Company: Premier Productions

Producer: R.J. Minney

Screenplay: Norman Lee, Harry Ostrer, Stafford Dickens (from a novel by Alfred Schirokauer)

Cinematography: Jack Cox

Editing: A.E. Bates

Art Direction: Albert Jullion

Costume Design: Honoria Plesch

Music: Mischa Spoliansky

Main cast: Michael Rennie (Hertz), Beryl Baxter (Theresa), Christine Norden (Cora Pearl), Miles Malleson (Offenbach), Andrew Osborn (Antoine), Andrew Cruickshank (Prince Nicholas), Margaretta Scott (Empress Eugenie), Kenneth Kent (Emperor Napoleon)

Saints and Sinners, 1949, 85 mins

Director: Leslie Arliss

Production Company: London Films

Producer: Arliss

Screenplay: Arliss, Paul Vincent Carroll

Cinematography: Osmond Borrodaile 
Editing: David Newhouse

Art Direction: Wilfred Shingleton

Costume Design: Honoria Plesch

Music: Philip Green

Main cast: Kieron Moore (Michael Kissane), Christine Norden (Blanche), Sheila Manahan (Sheila Flaherty), Michael Dolan (Canon), Maire O’Neill (Ma Flanagan), Tom Dillon (O’Brien), Pamela Arliss (secretary), Eddie Byrne (Norreys), Anita Bolster (Julia Ann Dermody)

The Woman's Angle, 1952, 86 mins

Director: Leslie Arliss

Production Company: Bow Bells Productions

Producer: Walter C. Mycroft

Screenplay: Arliss, Mabbie Poole (from a novel by Ruth Feiner)

Cinematography: Erwin Hillier

Editing: B. Jarvis

Art Direction: Terence Verity

Costume Design: Babs Gray

Music: Robert Gill, Louis Levy

Main cast: Edward Underdown (Robert Mansell), Cathy O'Donnell (Nina Van Rhyne), Lois Maxwell (Enid Mansell), Claude Farell (Delysia Veronova), Peter Reynolds (Brian Mansell), Marjorie Fielding (Mrs Mansell), Anthony Nicholls (Dr Jarvis), Isabel Dean (Isobel Mansell)

\section{The Triangle, 1953, 77 mins}

This film consists of three episodes which first appeared separately on television before being spliced together for cinema release.

Director: Leslie Arliss ('Priceless Pocket'; other episodes directed by Lance Comfort, Bernard Knowles)

Production Company: Douglas Fairbanks Productions

Producer: Douglas Fairbanks Jr

Screenplay: Guy Morgan (from a story by Geoffrey Wallace)

Art Direction: Norman Arnold

Music: Bretton Byrd

Main cast: James Hayter (Henry Popple), Muriel George (Martha Popple)

Thought to Kill, 1954, 77 mins

This film consists of three episodes which first appeared separately on television before being spliced together for cinema release. 
Director: Leslie Arliss ('Thought to Kill'; other episodes directed by Lawrence Huntington, Bernard Knowles)

Production Company: Douglas Fairbanks Productions

Producer: Douglas Fairbanks Jr

Screenplay: Selwyn Jones

Art Direction: Norman Arnold

Music: Allan Gray

Main cast: Ernest Thesiger (Burden, Sr), Eileen Moore (Isobel), Brian Worth (Camden)

\section{Destination Milan, 1954, 78 mins}

This film consists of three episodes which first appeared separately on television before being spliced together for cinema release.

Director: Leslie Arliss ('Lowland Fling); other episodes directed by Lawrence Huntington, John Gilling)

Production Company: Douglas Fairbanks Productions

Producer: Douglas Fairbanks Jr

Screenplay: Paul Vincent Carroll

Music: Bretton Byrd

Main cast: Cyril Cusack (Paddy O'Clafferty), Barbara Mullen (Miss Busbee), John Laurie (Walter McHarry)

See How They Run, 1955, 84 mins

Director: Leslie Arliss

Production Company: Winwell

Producers: Bill Luckwell, D.E.A. Winn

Screenplay: Arliss, Val Valentine, Roy Miller (from a play by Philip King)

Cinematography: Ken Talbot

Editing: Sam Simmonds

Art Direction: Ivan King

Music: John Bath

Main cast: Ronald Shiner (Wally Winton), Greta Gynt (Penelope Toop), James Hayter (Bishop of Lax), Wilfrid Hyde-White (Brig. Buskin), Dora Bryan (Isa), Richard Wattis (Rev. Lionel Toop), Viola Lyel (Miss Skilton)

Miss Tulip Stays the Night, 1955, 68 mins

Director: Leslie Arliss

Production Company: Bill Luckwell Productions

Producer: John O. Douglas, Bill Luckwell

Screenplay: Douglas, Luckwell, Jack Hulbert 
Cinematography: Kent Talbot

Editing: Sam Simmonds

Art Direction: Ivan King

Music: De Wolfe

Main cast: Diana Dors (Kate Dax), Patrick Holt (Andrew Dax), Cicely

Courtneidge (Miss Tulip), Jack Hulbert (P.C. Feathers), A.E. Matthews

(Mr Potts), Joss Ambler (Insp. Thorne)

\section{Arthur Crabtree}

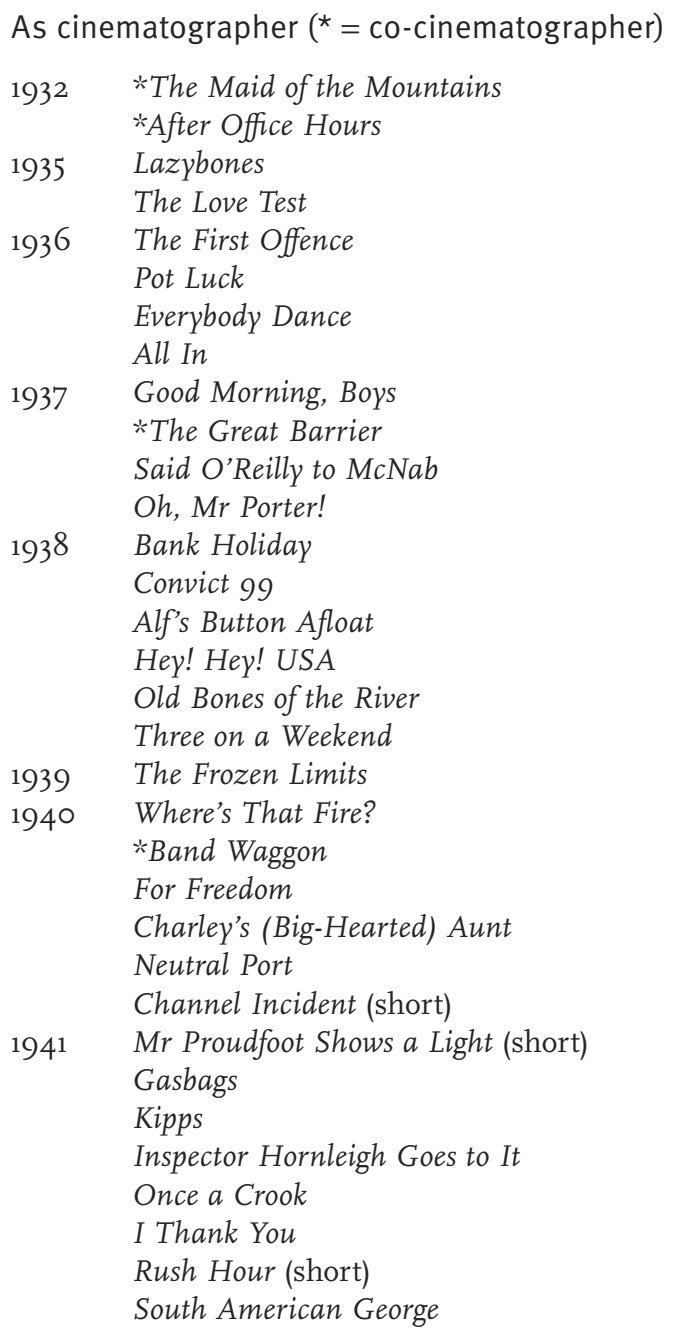




$\begin{array}{ll}1942 & \text { Uncensored } \\ & \text { Much Too Shy } \\ & \text { King Arthur Was a Gentleman } \\ 1943 & \text { The Man in Grey } \\ & \text { Dear Octopus } \\ 1944 & \text { Fanny by Gaslight } \\ 1945 & \text { Waterloo Road }\end{array}$

\section{As director}

Madonna of the Seven Moons, 1945, 110 mins

Director: Arthur Crabtree

Production Company: Gainsborough

Producer: R.J. Minney

Screenplay: Roland Pertwee, Brock Williams (from a novel by Margery Lawrence)

Cinematography: Jack Cox

Editing: Lito Carruthers

Art Direction: Andrew Mazzei

Costume Design: Elizabeth Haffenden

Music: Louis Levy

Main cast: Phyllis Calvert (Maddalena), Stewart Granger (Nino), Patricia Roc (Angela), Peter Glenville (Sandro), John Stuart (Giuseppe), Reginald Tate (Ackroyd), Peter Murray-Hill (Logan), Dulcie Gray (Nesta), Alan Haines (Evelyn), Jean Kent (Vittoria), Nancy Price (Mama Barucci), Amy Veness (Tessa), Hilda Bayley (Mrs Fiske), Robert Speaight (Priest), Helen Haye (Mother Superior), Danny Green (Scorpi)

They Were Sisters, 1945, 115 mins

Director: Arthur Crabtree

Production Company: Gainsborough

Producer: Harold Huth

Screenplay: Roland Pertwee, Katherine Strueby (from a novel by Dorothy Whipple)

Cinematography: Jack Cox

Editing: Charles Knott

Art Direction: David Rawnsley

Costume Design: Yvonne Caffin

Music: Hubert Bath, Louis Levy

Main cast: Phyllis Calvert (Lucy Moore), James Mason (Geoffrey Lee), Hugh Sinclair (Terry Crawford), Anne Crawford (Vera Sargeant), Dulcie Gray 
(Charlotte Lee), Peter Murray-Hill (William Moore), Barry Livesey (Brian Sargeant), Pamela Kellino (Margaret Lee), Ann Stephens (Judith Lee), Helen Stephens (Sarah Sargeant), Brian Nissen (John Watson), John Gilpin (Stephen Lee), David Horne (Mr Lee), Brefni O’Rourke (coroner)

Caravan, 1946, 122 mins

Director: Arthur Crabtree

Production Company: Gainsborough

Producer: Harold Huth

Screenplay: Roland Pertwee (from a novel by Eleanor Smith)

Cinematography: Stephen Dade, Cyril Knowles

Editing: Charles Knott

Art Direction: John Bryan

Costume Design: Elizabeth Haffenden

Music: Louis Levy

Main cast: Stewart Granger (Richard Darrell), Jean Kent (Rosal), Anne Crawford (Oriana Camperdene), Dennis Price (Sir Francis Castleton), Robert Helpmann (Wycroft), Gerard Hinze (Don Carlos), John Salew (Diego), Enid Stamp-Taylor (Bertha)

Dear Murderer, 1947, 94 mins

Director: Arthur Crabtree

Production Company: Gainsborough

Producer: Betty E. Box

Screenplay: Muriel and Sydney Box, Peter Rogers (from a play by St John Legh Clowes)

Cinematography: Stephen Dade

Editing: Gordon Hales

Art Direction: John Elphick

Costume Design: Yvonne Caffin

Music: Benjamin Frankel

Main cast: Eric Portman (Lee Warren), Greta Gynt (Vivien Warren), Dennis Price (Richard Fenton), Jack Warner (Insp. Penbury), Maxwell Reed (Jimmy Martin), Hazel Court (Avis Fenton), Jane Hylton (Rita), Andrew Crawford (Sgt Fox)

The Calendar, 1948, 80 mins

Director: Arthur Crabtree

Production Company: Gainsborough 
Producer: Antony Darnborough

Screenplay: Geoffrey Kerr (from a play by Edgar Wallace)

Cinematography: Reginald Wyer, Cyril Knowles

Editing: Charles Knott

Art Direction: John Elphick, George Provis

Costume Design: Julie Harris

Music: Arthur Wilkinson

Main cast: Greta Gynt (Wenda Panniford), John McCallum (Capt. Garry Anson), Raymond Lovell (Lord Willie Panniford), Sonia Holm (Lady Mollie Panniford), Leslie Dwyer (Sam Hillcott), Charles Victor (John Dory), Felix Aylmer (Lord Forlingham), Sidney King (Tony), Barry Jones (Sir John Garth), Diana Dors (Hawkins), Noel Howlett (lawyer)

Quartet, 1948, 120 mins

Director: Arthur Crabtree ('The Kite')

Production Company: Gainsborough

Producer: Antony Darnborough

Screenplay: R.C. Sherriff (from a story by W. Somerset Maugham)

Cinematography: Ray Elton

Editing: Charles Knott

Art Direction: Cedric Dawe

Costume Design: Julie Harris

Music: John Greenwood

Main cast: Mervyn Johns (Mr Swinley), Hermione Baddeley (Mrs Swinley), George Cole (Herbert), Susan Shaw (Betty), Bernard Lee (Prison Visitor), Frederick Leister (Prison Governor), David Cole (Herbert as a boy)

Don't Ever Leave Me, 1949, 85 mins

Director: Arthur Crabtree

Production Company: Triton

Producer: Betty E. Box

Screenplay: Robert Westerby (from a novel by Antony Armstrong)

Cinematography: Stephen Dade

Editing: Charles Knott

Art Direction: John Elphick

Costume Design: Joan Ellacott

Music: Lambert Williamson

Main cast: Jimmy Hanley (Jack Denton), Petula Clark (Sheila Farlaine), Linden Travers (Mary Lamont), Hugh Sinclair (Michael Farlaine), Edward Rigby (Harry Denton), Anthony Newley (Jimmy Knowles), Barbara Murray (Joan Robbins), Maurice Denham (Mr Knowles), Anthony Steel (Harris), Brenda Bruce (Miss Smith) 
Lilli Marlene, 1950, 85 mins

Director: Arthur Crabtree

Production Company: Monarch

Producer: William Gell

Screenplay: Leslie Wood

Cinematography: Jack Asher

Editing: Lister Laurance

Art Direction: R. Holmes Paul

Costume Design: Michael Whittaker

Music: Stanley Black

Main cast: Lisa Daniely (Lilli Marlene), Hugh McDermott (Steve), Richard

Murdoch (F/Lt Murdoch), Leslie Dwyer (Berry), Estelle Brody (herself),

Cecil Brock (O’Riley), John Blythe (Holt), Stanley Baker (Evans), Judith

Wharton (Auntie), Michael Ward (Wintertree)

Hindle Wakes, 1952, 88 mins

Director: Arthur Crabtree

Production Company: Monarch

Producer: William Gell

Screenplay: John Baines (from play by Stanley Houghton)

Cinematography: Geoffrey Faithfull

Editing: Max Benedict

Art Direction: Andrew Mazzei

Music: Stanley Black

Main cast: Lisa Daniely (Jenny Hawthorn), Brian Worth (Alan Jeffcote), Leslie Dwyer (Mr Hawthorn), Joan Hickson (Mrs Hawthorn), Sandra Dorne (Mary Hollins), Ronald Adam (Mr Jeffcote), Mary Clare (Mrs Jeffcote), Michael Medwin (George Ackroyd), Bill Travers (Bob), Beatrice Varley (Mrs Hollins)

The Wedding of Lilli Marlene, 1953, 89 mins

Director: Arthur Crabtree

Production Company: Monarch

Producer: William Gell

Screenplay: John Baines

Cinematography: Arthur Grant

Editing: Douglas Myers

Art Direction: Ray Simm

Music: Eric Rogers 
Main cast: Lisa Daniely (Lilli Marlene), Hugh McDermott (Steve Moray), Sidney James (Fennimore Hunt), Gabrielle Brune (Maggie Lennox), Jack Billings (Hal Marvel), Robert Ayres (Andrew Jackson), Joan Heal (Linda), Wally Patch (Wally), Rosie (Irene Handl)

Stryker of the Yard, 1953, 67 mins

Comprises two episodes of the television series: 'The Story of the Studio Payroll', 'Uncle Henry'.

Director: Arthur Crabtree

Production Company: Republic

Producer: William N. Boyle

Screenplay: Guy Morgan, Lester Powell

Cinematography: Basil Emmott

Editing: John Seabourne Sr

Music: Lambert Williamson

Main cast: Clifford Evans (Insp. Stryker), Jack Watling (Tony Ashworth), Susan Stephen (Peggy Sinclair), George Woodbridge (Sgt Walker), Eliot Wakeham (Henry Petheridge)

Morning Call, 1957, 75 mins

Director: Arthur Crabtree

Production Company: Winwell

Producer: Alfred Strauss

Screenplay: Paul Tabori, Bill Luckwell, Tom Waldron

Cinematography: James Harvey

Editing: John Ferris

Art Direction: John Stoll

Music: John Bath

Main cast: Greta Gynt (Annette Manning), Ron Randell (Nick Logan), Bruce Seton (Insp. Brown), Virginia Keiley (Vera Clark)

West of Suez, 1957, 85 mins

Director: Arthur Crabtree

Production Company: Winwell

Producer: Kay Luckwell, Derek Winn

Screenplay: Norman Hudis

Cinematography: James Harvey

Editing: Peter Mayhew 
Art Direction: John Stoll

Music: Wilfred Burns

Main cast: Keefe Brasselle (Brett Manders), Kay Callard (Pat), Karel Stepanek (Langford), Ursula Howells (Eileen), Bruce Seton (Major Osborne), Harry Fowler (Tommy)

Death over My Shoulder, 1958, 89 mins

Director: Arthur Crabtree

Production Company: Vicar

Producer: Frank Bevis

Screenplay: Norman Hudis, Alyce Canfield

Cinematography: James Harvey

Editing: Seymour Logie

Art Direction: Herbert Smith

Music: Douglas Gamley

Main cast: Keefe Brasselle (Jack Regan), Bonar Colleano (Jo Longo), Jill Adams (Evelyn Connors), Sonia Dresdel (Miss Upton), Arlene de Marco (Julie), Charles Farrell (Chiv)

Fiend Without a Face, 1958, 74 mins

Director: Arthur Crabtree

Production Company: Producers Associates

Producer: John Croydon

Screenplay: Herbert J. Leder (from a story by Amelia Reynolds Long)

Cinematography: Lionel Banes

Special Effects: Ruppel and Nordhoff, Peter Neilson

Editing: R.Q. McNaughton

Art Direction: John Elphick

Music: Buxton Orr

Main cast: Marshall Thompson (Major Jeff Cummings), Terence Kilburn

(Capt. Al Chester), Stanley Maxted (Col. Butler), Kim Parker (Barbara Griselle), Kynaston Reeves (Professor Walgate)

Horrors of the Black Museum, 1959, 80 mins

Director: Arthur Crabtree

Production Company: Anglo-Amalgamated

Producer: Jack Greenwood, Herman Cohen

Screenplay: Cohen, Aben Kandel

Cinematography: Desmond Dickinson 


\section{FILMOGRAPHIES}

Editing: Geoffrey Muller

Art Direction: Wilfred Arnold

Costume Design: Anna Duse

Music: Gerard Schurmann

Main cast: Michael Gough (Edmond Bancroft), Graham Curnow (Rick), June Cunningham (Joan), Shirley Anne Field (Angela), Geoffrey Keen (Supt Graham), John Warwick (Insp. Lodge), Beatrice Varley (Aggie), Gerald Anderson (Dr Ballan)

\section{Bernard Knowles}

\section{As cinematographer}

$\begin{array}{ll}1927 & \text { Mumsie } \\ 1928 & \text { Dawn } \\ & \text { Love's Option } \\ 1929 & \text { The Broken Melody } \\ & \text { Auld Lang Syne } \\ & \text { The Silver King } \\ 1930 & \text { Rookery Nook } \\ & \text { French Leave } \\ & \text { School for Scandal } \\ & \text { Canaries Sometimes Sing } \\ 1931 & \text { The Calendar } \\ 1932 & \text { The Hound of the Baskervilles } \\ & \text { White Face } \\ 1933 & \text { The Good Companions } \\ & \text { Falling for You } \\ 1934 & \text { Jack Ahoy! } \\ & \text { The Camels Are Coming } \\ & \text { Jew Süss (lighting technician) } \\ & \text { Forever England } \\ 1935 & \text { The 39 Steps } \\ & \text { King of the Damned } \\ 1939 & \text { Rhodes of Africa } \\ & \text { Secret Agent } \\ & \text { East Meets West } \\ & \text { Sabotage } \\ & \text { Take My Tip } \\ & \text { French Witho Ind Innocent } \\ & \text { Spy for a Day } \\ & \text { Gaslight } \\ & \end{array}$


1941 Freedom Radio

Quiet Wedding

The Saint's Vacation

Jeannie

1942 The Day Will Dawn (uncredited)

Unpublished Story

Secret Mission

Talk About Jacqueline

1943 The Demi-Paradise

1944 English Without Tears

Love Story

\section{As director}

A Place of One's Own, 1945, 92 mins

Director: Bernard Knowles

Production Company: Gainsborough

Producer: R.J. Minney

Screenplay: Brock Williams (from a novel by Osbert Sitwell)

Cinematography: Stephen Dade

Editing: Charles Knott

Art Direction: John Elphick

Costume Design: Elizabeth Haffenden

Music: Hubert Bath

Main cast: Margaret Lockwood (Annette), James Mason (Mr Smedhurst), Barbara Mullen (Mrs Smedhurst), Dennis Price (Dr Selbie), Helen Haye (Mrs Manning Tutthorn), Michael Shepley (Major Manning Tutthorn), Dulcie Gray (Sarah), Ernest Thesiger (Dr Marsham), Moore Marriott (George), O.B. Clarence (Perkins), Edie Martin (Cook), Muriel George (Nurse)

The Magic Bow, 1946, 106 mins

Director: Bernard Knowles

Production Company: Gainsborough

Producer: R.J. Minney

Screenplay: Roland Pertwee (from a novel by Manuel Komroff)

Cinematography: Jack Cox

Editing: Alfred Roome

Art Direction: Andrew Mazzei

Costume Design: Elizabeth Haffenden

Music: Louis Levy 
Main cast: Stewart Granger (Nicolo Paganini), Phyllis Calvert (Jeanne de Vermond), Jean Kent (Bianci), Dennis Price (Paul de la Rochelle), Cecil Parker (Luigi Germi), Felix Aylmer (Signor Fazzini), Frank Cellier (Antonio), Marie Lohr (Countess de Vermond), Robert Speaight (Cardinal), Mary Jerrold (Teresa Paganini), David Horne (Rizzi)

The Man Within, 1947, 90 mins

Director: Bernard Knowles

Production Company: A Sydney Box Production/Production Film Service Producers: Sydney and Muriel Box

Screenplay: Sydney and Muriel Box

Cinematography: Geoffrey Unsworth

Editing: Alfred Roome

Art Direction: Andrew Mazzei

Costume Design: Elizabeth Haffenden

Music: Clifton Parker

Main cast: Michael Redgrave (Richard Carlyon), Richard Attenborough

(Francis Andrews), Jean Kent (Lucy), Joan Greenwood (Elizabeth), Francis L. Sullivan (Mr Braddock), Felix Aylmer (Priest), Ronald Shiner (Cockney Harry), Basil Sydney (Sir Henry Merriman), Ernest Thesiger (Farne), Allan Jeayes (Judge), Ralph Truman (Prison Interrogator)

Jassy, 1947, 102 mins

Director: Bernard Knowles

Production Company: Gainsborough

Producer: Sydney Box

Screenplay: Dorothy and Campbell Christie, Geoffrey Kerr (from a novel by Norah Lofts)

Cinematography: Geoffrey Unsworth

Editing: Charles Knott

Art Direction: Maurice Carter

Costume Design: Elizabeth Haffenden

Music: Louis Levy

Main cast: Margaret Lockwood (Jassy Woodroofe), Patricia Roc (Dilys Helmar), Dennis Price (Christopher Hatton), Basil Sydney (Nick Helmar), Dermot Walsh (Barney Hatton), Linden Travers (Beatrice Helmar), Esma Cannon (Lindy Wicks), Cathleen Nesbitt (Elizabeth Twisdale), Nora Swinburne (Mrs Hatton), Jean Cadell (Meggie), John Laurie (Tom Woodroofe), Grey Blake (Stephen Fennell), Bryan Coleman (Sedley), Torin Thatcher (Wicks), Beatrice Varley (Mrs Wicks) 
The White Unicorn, 1947, 97 mins

Director: Bernard Knowles

Production Company: John Corfield Productions

Producer: Harold Huth

Screenplay: Robert Westerby, A.R. Rawlinson, Moie Charles

Cinematography: Reginald Wyer

Art Direction: Norman Arnold

Costume Design: Mattli

Music: Bretton Byrd

Main cast: Margaret Lockwood (Lucy), Joan Greenwood (Lottie Smith), Ian Hunter (Philip Templar), Dennis Price (Richard Glover), Joan (Eileen Peel), Guy Middleton (Fobey), Catherine Lacey (Miss Cater), Paul Dupuis (Paul), Valentine Dyall (Storton), Julia Lockwood (Norey)

\section{Easy Money, 1948, 94 mins}

Director: Bernard Knowles

Production Company: Gainsborough

Producer: A. Frank Bundy

Screenplay: Muriel and Sydney Box (from a play by Arnold Ridley)

Cinematography: Jack Asher

Editing: V. Sagovsky

Art Direction: Cedric Dawe

Costume Design: Joan Ellacott

Music: Temple Abady

Main cast: Jack Warner (Philip Stafford), Marjorie Fielding (Ruth Stafford), Petula Clark (Jackie), David Tomlinson (Martin), Yvonne Owen (Carol), Jack Watling (Dennis), Mervyn Johns (Atkins), Joan Young (Agnes), Greta Gynt (Pat), Dennis Price (Joe), Bill Owen (Lee), Edward Rigby (Teddy), Guy Rolfe (Archie), Raymond Lovell (conductor), Frank Cellier (manager)

The Perfect Woman, 1949, 89 mins

Director: Bernard Knowles

Production Company: Two Cities

Producer: George and Alfred Black

Screenplay: Knowles, George Black, J.B. Boothroyd (from a play by Wallace Geoffrey, Basil Mitchell)

Cinematography: Jack Hildyard

Editing: Peter Graham Scott

Art Direction: J. Elder Wills

Music: Arthur Wilkinson 
Main cast: Patricia Roc (Penelope Belman), Nigel Patrick (Roger Cavendish), Stanley Holloway (Ramshead), Miles Malleson (Prof. Ernest Belman), Irene Handl (Mrs Butters), Pamela Devis (Olga the robot), Anita Bolster (Lady Diana), Dora Bryan (model), Fred Berger (Farini), David Hurst (Wolfgang Winkel)

The Lost People, 1949, 88 mins

Director: Bernard Knowles

Production Company: Gainsborough

Producer: Gordon Wellesley

Screenplay: Bridget Boland (from her play)

Cinematography: Jack Asher

Editing: Gordon Hale

Art Direction: John Elphick

Costume Design: Julie Harris

Music: John Greenwood

Main cast: Dennis Price (Ridley), Mai Zetterling (Lily), Richard Attenborough (Jan), Siobhan McKenna (Marie), Maxwell Reed (Peter), William Hartnell (Barnes), Gerard Heinz (Professor), Zena Marshall (Anna) Olaf Pooley (Milosh), Harcourt Williams (Priest), Jill Balcon (Rebecca)

The Reluctant Widow, 1950, 91 mins

Director: Bernard Knowles

Production Company: Two Cities

Producer: Gordon Wellesley

Screenplay: Wellesley, J.B. Boothroyd (from a novel by Georgette Heyer)

Cinematography: Jack Hildyard

Editing: John D. Guthridge

Art Direction: Carmen Dillon

Costume Design: Beatrice Dawson

Music: Allan Gray

Main cast: Jean Kent (Helena), Guy Rolfe (Lord Carlyon), Paul Dupuis (Lord Nivelle), Lana Morris (Becky), Kathleen Byron (Annette de Chevreaux), Julian Dallas (Francis Cheviot), Peter Hammond (Eustace Cheviot), Anthony Tancred (Nicky), Jean Cadell (Mrs Barrows), Andrew Cruickshank (Lord Bedlington), George Thorpe (Col. Strong)

Park Plaza 605, 1953, 75 mins

Director: Bernard Knowles

Production Company: B \& A

Producers: Albert Fennell, Bertram Ostrer 
Screenplay: Knowles, Fennell, Ostrer

Editing: Clifford Boote

Art Direction: John Elphick

Costume Design: Elsa Fennell

Music: Philip Green

Main cast: Tom Conway (Norman Conquest), Eva Bartok (Nadine Rodin), Joy Shelton (Pixie Everard), Sidney James (Supt Williams), Michael Balfour (Ted Birston), Richard Wattis (Theodore Feather), Carl Jaffe (Boris Roff), Anton Diffring (Gregor)

\section{The Triangle, 1953, 77 mins}

This film consists of three episodes which first appeared separately on television before being spliced together for cinema release.

Director: Bernard Knowles ('A Lodging for the Night'; other episodes directed by Lance Comfort, Leslie Arliss)

Production Company: Douglas Fairbanks Productions

Producer: Douglas Fairbanks Jr

Screenplay: Larry Marcus (from a story by Robert Louis Stevenson)

Art Direction: Norman Arnold

Main cast: Douglas Fairbanks Jr (François Villon), Felix Aylmer (Brisetout)

\section{Thought to Kill, 1954, 77 mins}

This film consists of three episodes which first appeared separately on television before being spliced together for cinema release.

Director: Bernard Knowles ('The Five Pound Note'; other episodes directed by Leslie Arliss, Lawrence Huntington)

Production Company: Douglas Fairbanks Productions

Producer: Douglas Fairbanks Jr

Screenplay: Robert Westerby, Larry Marcus

Cinematography: Eric Cross

Art Direction: Norman Arnold

Music: Allan Gray

Main cast: Ernest Thesiger (Burden, Sr), Eileen Moore (Isobel), Brian Worth (Camden)

Handcuffs, London, 1955, 76 mins

Director: Bernard Knowles

Production Company: Trinity

Producer: John Larkin

Screenplay: Brock Williams 
Cinematography: Brendan Stafford

Editing: Peter Seabourne

Art Direction: Norman Arnold

Music: Eric Spear

Main cast: Bruce Seton (Supt Robert Fabian), Kathleen Byron (Janet Tedford), Michael Craig (Roger Garven), Isobel Dean (Doris Tedford), Alexander Gauge (Nicholas Bardwill), Ursula Howells (Madelaine Perry)

Barbados Quest, 1955, 70 mins

Director: Bernard Knowles

Production Company: Cipa

Producers: Robert S. Baker, Monty Berman

Screenplay: Kenneth R. Hayles

Cinematography: Monty Berman

Editing: Jack Slade

Art Direction: Wilfred Arnold

Main cast: Tom Conway (Tom Martin), Delphi Lawrence (Jean Larson), Brian Worth (Geoffrey Blake), Michael Balfour (Barney Wilson), Campbell Cotts (Robert Coburn), John Horsley (Det. Insp. Taylor), Grace Arnold (Lady Hawksley), Ronan O’Casey (Stefan Gordoni)

Frozen Alive, 1964, 80 mins (UK/West Germany)

Director: Bernard Knowles

Production Company: Alfa, Creole

Producers: Arthur Brauner, Ronald Rietti

Screenplay: Evelyn Frazer

Cinematography: Robert Ziller

Editing: Steve Collins

Art Direction: Jürgen Klebach

Costume Design: Vera Mügge

Music: Eric Spear

Main cast: Mark Stevens (Dr Frank Overton), Marianne Koch (Dr Helen Wieland), Wolfgang Lukschy (Insp. Prenton), Delphi Lawrence (Joan Overton), John Longden (Prof. Hubbard)

Spaceflight IC-I: An Adventure in Space, 1965, 65 mins

Director: Bernard Knowles

Production Company: Lippert Films

Producers: Robert J. Lippert, Jack Parsons

Screenplay: Henry Cross 
Cinematography: Geoffrey Faithfull

Editing: Robert Winter

Art Direction: Harry White

Costume Design: Jean Fairlie

Music: Elizabeth Lutyens

Main cast: Bill Williams (Capt. Mead Ralston), Kathleen Breck (Kate

Saunders), John Cairney (Dr Steven Thomas), Donald Churchill (Carl

Walcott), Linda Marlowe (Dr Helen Thomas)

Hell Is Empty, 1967, 109 mins

Director: Bernard Knowles

Production Company: Dominion

Producer: Michael Eland, Ronald Rietti

Screenplay: Knowles, John Ainsworth, John Fowler

Editing: Jim Connock

Music: Georges Garvarentz

Main cast: Martine Carol (Martine Grant), Anthony Steel (Major Morton), James Robertson Justice (Angus McGee), Shirley Anne Field (Shirley McGee), Isa Miranda (Isa Grant)

\section{Lawrence Huntington}

\begin{tabular}{|c|c|}
\hline 1939 & I Killed the Count (with Alec Coppel) \\
\hline 1941 & Sheepdog of the Hills \\
\hline 1951 & One Wild Oat (with Vernon Sylvaine) \\
\hline 1952 & Deadly Nightshade \\
\hline 1961 & A Question of Suspense \\
\hline 1969 & The Oblong Box \\
\hline
\end{tabular}

As producer only

1935 Lieutenant Daring R.N.

1961 The Trunk

As director (other functions as noted)

After Many Years, 1930, 70 mins

Director: Lawrence Huntington

Production Company: Savana 
Producer: Alvin Saxon

Screenplay: Huntington

Main cast: Henry Thompson, Nancy Kenyon, Savoy Havana Band

Romance in Rhythm, 1935, 73 mins

Director: Lawrence Huntington

Production Company: Allied Film Productions

Producer: Huntington

Screenplay: Huntington

Cinematography: Desmond Dickinson

Editing: Challis Sanderson

Main cast: Phyllis Clare (Ruth Lee), David Hutcheson (Bob Mervyn)

Strange Cargo, 1936, 68 mins

Director: Lawrence Huntington

Production Company: British \& Dominions

Producer: Huntington

Screenplay: Gerald Elliott

Cinematography: Francis Carver

Main cast: Kathleen Kelly (Sonia), George Mozart ('Orace), George Sanders (Roddy)

Two on a Doorstep, 1936, 71 mins

Director: Lawrence Huntington

Production Company: British \& Dominions

Producer: Anthony Havelock-Allan

Screenplay: Gerald Elliott, George Baraud

Cinematography: Francis Carver

Main cast: Kay Hammond (Jill Day), Harold French (Jimmy Blair), George Mozart (George)

Café Mascot, 1936, 77 mins

Director: Lawrence Huntington

Production Company: Pascal Films

Producer: Gabriel Pascal

Screenplay: Gerald Elliott 
Cinematography: Stanley Grant

Main cast: Geraldine Fitzgerald (Moira O’Flynn), Derrick de Marney (Jerry Wilson)

Full Speed Ahead, 1936, 71 mins

Director: Lawrence Huntington

Production Company: Lawrence Huntington Productions

Producer: Huntington

Screenplay: Huntington, Gerald Elliott

Cinematography: Stanley Grant

Main cast: Paul Murton (Capt. Murton), Moira Lynd (Jean), George Mozart (Chief Smith)

The Bank Messenger Mystery, 1936, 57 mins

Director: Lawrence Huntington

Production Company: Hammer

Producer: Will Hammer

Screenplay: Huntington

Cinematography: Eric Cross

Main cast: George Mozart (George Brown), Paul Neville (Harper)

Screen Struck, 1937, 38 mins

Director: Lawrence Huntington

Production Company: U.K. Films

Producers: John Barter, John Baxter

Screenplay: Herbert Ayres

Cinematography: Jack Parker

Main cast: Julian Vedey (Al Sugarman), Diana Beaumont (Marjorie)

Twin Faces, 1937, 67 mins

Director: Lawrence Huntington

Production Company: Premier Sound Films

Producer: J. Steven Edwards

Screenplay: Gerald Elliott

Main cast: Anthony Ireland (Jimmy Strangeways), Francesca Bahrle (Judy

Strangeways) 
222 FILMOGRAPHIES

Passenger to London, 1937, 57 mins

Director: Lawrence Huntington

Production Company: Fox-British

Producer: Huntington

Screenplay: David Evans

Cinematography: Stanley Grant

Editing: Peter Tanner

Art Direction: William Hemsley

Music: Charles Cowlrick

Main cast: John Warwick (Frank Drayton), Jenny Laird (Barbara Lane), Paul Neville (Vautel)

Dial 999, 1937, 46 mins

Director: Lawrence Huntington

Production Company: Fox-British

Producer: Edward Dryhurst

Screenplay: Ernest Dudley

Cinematography: Stanley Grant

Editing: Peter Tanner

Art Direction: William Hemsley

Main cast: John Longden (Bill Waring), Elizabeth Kent (Margot Curtis),

Neville Brook (Hicks)

Bad Boy, 1938, 69 mins

Director: Lawrence Huntington

Production Company: Radius Films

Producer: Vaughan N. Dean

Screenplay: Huntington

Cinematography: Stanley Grant

Main cast: John Warwick (Nick Bryan) John Longden (Insp. Bryan), Kathleen

Kelly (Ann Travers)

The Patient Vanishes/This Man Is Dangerous, 1941, 82 mins

Director: Lawrence Huntington

Production Company: Rialto

Producer: John Argyle

Screenplay: Argyle, Edward Dryhurst (from a novel by David Hume)

Cinematography: Bryan Langley 
Music: Guy Jones

Main cast: James Mason (Mick Cardby), Mary Clare (Matron), Margaret Vyner (Molly Bennett), Gordon McLeod (Insp. Cardby), Barbara Everest (Mrs Cardby)

Tower of Terror, 1941, 78 mins

Director: Lawrence Huntington

Production Company: Associated British

Producer: John Argyle

Screenplay: Argyle, John Reinhardt

Cinematography: Walter J. Harvey, Ronald Anscombe

Editing: Flora Newton

Art Direction: J. Charles Gilbert

Music: Charles Williams

Main cast: Wilfrid Lawson (Wolfe Kristan), Michael Rennie (Anthony Hale), Movita (Marie Durand), Morland Graham (Kleber)

Suspected Person, 1942, 75 mins

Director: Lawrence Huntington

Production Company: Associated British

Producer: Warwick Ward

Screenplay: Huntington

Cinematography: Günther Krampf

Editing: Flora Newton

Music: Guy Jones

Main cast: Clifford Evans (Jim Raynor), Patricia Roc (Joan Raynor), David

Farrar (Insp. Thompson), Anne Firth (Carol), Robert Beatty (Franklin)

Women Aren't Angels, 1942, 85 mins

Director: Lawrence Huntington

Production Company: Associated British

Producer: Warwick Ward

Screenplay: Huntington, Bernard Mainwaring, Vernon Sylvaine (from Sylvaine's play)

Cinematography: Günther Krampf

Music: Charles Williams

Main cast: Robertson Hare (Wilmer Popday), Alfred Drayton (Alfred Bandle), Joyce Heron (Thelma), Mary Hinton (Thelma Bandle), Peggy Novak (Elizabeth Popday) 
Warn That Man, 1943, 82 mins

Director: Lawrence Huntington

Production Company: Associated British

Producer: Warwick Ward

Screenplay: Huntington, Vernon Sylvaine (from Sylvaine's play)

Cinematography: Günther Krampf

Music: Charles Williams

Main cast: Raymond Lovell (Lord Buckley/Hausemann), Finlay Currie (Capt. Fletcher), Philip Friend (John Cooper), Jean Kent (Frances Lane), Frederick Cooper (Charles/Frampton)

Night Boat to Dublin, 1946, 99 mins

Director: Lawrence Huntington

Production Company: Associated British

Producer: Hamilton G. Inglis

Screenplay: Huntington, Robert Hall

Cinematography: Otto Heller

Editing: Flora Newton

Art Direction: David Rawnsley

Costume Design: Anna Duse

Music: Charles Williams

Main cast: Robert Newton (Capt. David Grant), Raymond Lovell (Paul Faber), Muriel Pavlow (Marion Decker), Guy Middleton (Capt. Toby Hunter), Herbert Lom (Keitel), John Ruddock (Bowman), Martin Miller (Prof. Hansen), Brenda Bruce (Lily Leggett), Leslie Dwyer (George Leggett), Julian Dallas (Lt Allen), Marius Goring (Jannings), Olga Lindo (Mrs Coleman), Stuart Lindsell (Insp. Martin), Valentine Dyall (Sir George Bell)

Wanted for Murder, 1946, 103 mins

Director: Lawrence Huntington

Production Company: Excelsior

Producer: Marcel Hellman

Screenplay: Emeric Pressburger, Rodney Ackland (from a play by Percy Robinson and Terence de Marney)

Cinematography: Max Greene

Editing: E.B. Jarvis

Art Direction: Charles Gilbert

Costume Design: Anna Duse

Music: Mischa Spoliansky 
Main cast: Eric Portman (Victor Coleman), Dulcie Gray (Anne Fielding), Derek Farr (Jack Williams), Roland Culver (Insp. Conway), Stanley Holloway (Sgt Sullivan), Barbara Everest (Mrs Coleman), Bonar Colleano (Cpl Mappolo), Jenny Laird (Jeannie), Kathleen Harrison (Florrie), Viola Lyel (Mrs Cooper), Bill Shine (Det. Ellis)

The Upturned Glass, 1947, 86 mins

Director: Lawrence Huntington

Production Company: Triton

Producers: Sydney Box, James Mason, Betty E. Box

Screenplay: Jno P. Monaghan, Pamela Kellino

Cinematography: Reginald Wyer

Editing: Alan Osbiston

Art Direction: Andrew Mazzei

Costume Design: Mattli

Music: Bernard Stevens

Main cast: James Mason (Michael Joyce), Rosamund John (Emma Wright), Pamela Kellino (Kate Howard), Brefni O’Rourke (Dr Farrell), Morland Graham (Clay), Ann Stephens (Ann Wright), Henry Oscar (Coroner), Jane Hylton (Miss Marsh)

When the Bough Breaks, 1947, 81 mins

Director: Lawrence Huntington

Production Company: Gainsborough

Producer: Betty E. Box, Antony Darnborough

Screenplay: Muriel and Sydney Box, Peter Rogers

Cinematography: Bryan Langley

Editing: Gordon Hales

Art Direction: John Elphick

Costume Design: Yvonne Caffin

Music: Clifton Parker

Main cast: Patricia Roc (Lily Bates), Rosamund John (Frances Norman), Bill Owen (Bill Collins), Cavan Malone (Jimmy), Patrick Holt (Robert Norman), Brenda Bruce (Ruby Chapman), Leslie Dwyer (George), Catherine Lacey (almoner), Muriel George ( $1^{\text {st }}$ landlady), Torin Thatcher (Adams), Edith Sharpe (Matron), Jane Hylton (maid)

Mr Perrin and Mr Traill, 1948, 92 mins

Director: Lawrence Huntington

Production Company: Two Cities 
Producer: Alexander Galperson

Screenplay: L.A.G. Strong (from a novel by Hugh Walpole)

Cinematography: Erwin Hillier

Editing: Ralph Kempton

Art Direction: Tom Morahan

Music: Allan Gray

Main cast: David Farrar (David Traill), Marius Goring (Vincent Perrin), Greta Gynt (Isabel Lester), Raymond Huntley (Moy-Thompson), Edward Chapman (Birkland), Mary Jerrold (Mrs Perrin), Ralph Truman (Comber), Finlay Currie (Sir Joshua Varley)

Man on the Run, 1949, 82 mins

Director: Lawrence Huntington

Production Company: Associated British

Producer: Huntington

Screenplay: Huntington

Cinematography: Wilkie Cooper

Editing: Monica Kimick

Art Direction: Terence Verity

Costume Design: Peggy Henderson

Music: Philip Green

Main cast: Derek Farr (Sgt Peter Burden, aka Brown), Joan Hopkins (Jean Adams), Edward Chapman (Insp. Mitchell), Laurence Harvey (Det. Sgt Lawson), John Stuart (Det. Insp. McBane), Howard Marion-Crawford ( $1^{\text {st }}$ paratrooper), Kenneth More (Cpl Newman), Eleanor Summerfield (May Baker), Valentine Dyall (Army Judge Advocate)

The Franchise Affair, 1951, 88 mins

Director: Lawrence Huntington

Production Company: Associated British

Producer: Robert Hall

Screenplay: Huntington, Robert Hall

Cinematography: Günther Krampf

Editing: Clifford Boote

Art Direction: Terence Verity

Music: Philip Green

Main cast: Michael Denison (Robert Blair), Dulcie Gray (Marion Sharpe), Marjorie Fielding (Mrs Sharpe), Anthony Nicholls (Kevin MacDermott), Athene Seyler (Aunt Lin), Ann Stephens (Betty Kane), Hy Hazell (Mrs Chadwick), John Bailey (Insp. Grant), Kenneth More (Stanley), Avice Landone (Mrs Wynn), Peter Jones (Bernard Chadwick) 
There Was a Young Lady, 1953, 70 mins

Director: Lawrence Huntington

Production Company: Nettlefold

Producer: A.R. Rawlinson

Screenplay: Huntington (from a radio serial by Vernon Harris, John Jowett) Cinematography: Gerald Gibbs

Editing: Joseph Sterling

Art Direction: Fred Pusey

Costume Design: June Kirby

Music: Wilfred Burns

Main cast: Dulcie Gray (Elizabeth Foster), Michael Denison (David Walsh),

Sydney Tafler (Johnny), Charles Farrell (Arthur), Bill Owen (Joe), Robert Adair (Basher), Geraldine McEwan (Irene)

\section{The Accused, 1953, 52 mins}

This film consists of two episodes prepared for and shown on television before being spliced together for cinema release.

Director: Lawrence Huntington (for episode 'The Accused'; other episode by Charles Saunders)

Production Company: Douglas Fairbanks Productions

Producer: Douglas Fairbanks Jr

Screenplay: John and Gwen Bagni

Cinematography: Gerry Gibbs

Editing: Joseph Sterling

Music: Allan Gray

Main cast: Clifford Evans (Dan Anderson), Mary Laura Wood (Edna), Jean Lodge (Sheila)

The Genie, 1953, 75 mins

This film consists of three episodes which first appeared separately on television before being spliced together for cinema release.

Director: Lawrence Huntington (for episode 'Emerald Green'; others directed by Lance Comfort)

Production Company: Douglas Fairbanks Productions

Producer: Douglas Fairbanks Jr

Screenplay: Paul Vincent Carroll

Cinematography: Eric Cross

Editing: Peter Taylor

Art Direction: Norman G. Arnold

Music: Allan Gray 
Main cast: Tommy Duggan (Mike), Bernadette O’Farrell (Molly), Sean Barrett (Brian)

Thought to Kill, 1954, 77 mins

This film consists of three episodes which first appeared separately on television before being spliced together for cinema release.

Director: Lawrence Huntington ('The Parlour Trick'; other episodes directed by Leslie Arliss, Bernard Knowles)

Production Company: Douglas Fairbanks Productions

Producer: Douglas Fairbanks Jr

Screenplay: Roland Pertwee

Art Direction: Norman Arnold

Music: Bretton Byrd

Main cast: Bill Owen (Arthur Preece), Avice Landone (Maud), Lana Morris (Ruby)

The Red Dress, 1954, 76 mins

This film consists of three episodes which first appeared separately on television before being spliced together for cinema release.

Director: Lawrence Huntington ('The Red Dress' and 'Panic'; the other by Charles Saunders)

Production Company: Douglas Fairbanks Productions

Producer: Douglas Fairbanks Jr

Screenplay: Guy Morgan ('The Red Dress', from a story by Rhys Davies); Larry Marcus, Peter Quinn ('Panic')

Cinematography: Ken Talbot, Jimmy Wilson

Editing: Sam Simmonds, Peter Taylor

Art Direction: Norman G. Arnold

Music: Allan Gray

Main cast: 'The Red Dress' - Renée Asherson (Megan), Clifford Evans (Sam); 'Panic' - James Kenney (Fred), John Salew (Karl)

Destination Milan, 1954, 78 mins

This film consists of three episodes which first appeared separately on television before being spliced together for cinema release.

Director: Lawrence Huntington (other episodes directed by Leslie Arliss, John Gilling) 
Producer: Tom D. Connochie

Production Company: Douglas Fairbanks Productions

Screenplay: Huntington, Robert Hall

Cinematography: Jimmy Wilson

Editing: Peter Taylor

Art Direction: Norman G. Arnold

Music: Allan Gray

Main cast: Tom Duggan (Leonard), Lorraine Clewes (Arlette), Christopher Lee (Svenson)

Contraband Spain, 1955, 82 mins

Director: Lawrence Huntington

Production Company: Diadem

Producer: Ernest Gartside

Screenplay: Huntington

Cinematography: Harry Waxman

Editing: Tom Simpson

Art Direction: Cedric Dawe

Music: Edwin Astley

Main cast: Richard Greene (Lee Scott), Michael Denison (Ricky Metcalfe), Anouk Aimée (Elena Vargas)

Deadly Record, 1959, 58 mins

Director: Lawrence Huntington

Production Company: Independent Artists

Producer: Vivian A. Cox

Screenplay: Huntington, Cox

Cinematography: Eric Cross

Editing: Eric Boyd-Perkins

Art Direction: Harry Pottle

Music: Neville McGrah

Main cast: Lee Patterson (Trevor Hamilton), Barbara Shelley (Susan Webb),

Jane Hylton (Ann Garfield), Geoffrey Keen (Supt Ambrose)

The Fur Collar, 1962, 71 mins

Director: Lawrence Huntington

Production Company: Albatross 
Producer: Huntington

Screenplay: Huntington

Cinematography: S.D. Onions

Editing: Peter Weatherley

Art Direction: Robert Jones

Music: John Fox

Main cast: John Bentley (Mike Andrews), Philip Friend (Eddie Morgan), Nadja Rejin (Marie Lejeune)

Stranglehold, 1962, 73 mins

Director: Lawrence Huntington

Production Company: Argo

Producers: Jack Lamont, David Henley

Screenplay: Guy Elmes

Cinematography: S.D. Onions

Editing: Peter Weatherley

Art Direction: Duncan Sutherland

Costume Design: Eve Faloon

Music: Eric Spear

Main cast: Macdonald Carey (Bill Morrison), Barbara Shelley (Chris Morrison), Philip Friend (Steffan), Nadja Rejin (Lilli)

Death Drums Along the River, 1963, 83 mins

Director: Lawrence Huntington

Production Company: Planet

Producer: Harry Alan Towers

Screenplay: Towers, Nicolas Roeg (from a novel by Edgar Wallace)

Cinematography: Bob Huke

Editing: Alan Morrison

Costume Design: Mary McFadden

Music: Sidney Torch

Main cast: Richard Todd (Insp. Harry Sanders), Marianne Koch (Dr Inge Jung), Albert Lieven (Dr Franz Weiss), Walter Rilla (Dr Schneider), Robert Arden (Jim Hunter), Jeremy Lloyd (Tom Hamilton)

The Vulture, 1967, 91 mins

Director: Lawrence Huntington

Production Companies: Homeric, Iliad, Film Financial Co. 
Producer: Huntington

Screenplay: Huntington

Cinematography: Stephen Dade

Editing: John S. Smith

Art Direction: Duncan Sutherland

Costume Design: Dulcie Midwinter

Music: Eric Spear

Main cast: Robert Hutton (Dr Eric Lutens), Akim Tamiroff (Prof. Hans Koniglich), Broderick Crawford (Brian Stroud), Diane Clare (Trudy Lutens), Philip Friend (Vicar) 